\title{
Targeting of Ikaros to pericentromeric heterochromatin by direct DNA binding
}

\author{
Bradley S. Cobb, ${ }^{1}$ Susana Morales-Alcelay, ${ }^{3}$ Gary Kleiger, ${ }^{2}$ Karen E. Brown, ${ }^{3}$ Amanda G. Fisher, ${ }^{3}$ \\ and Stephen T. Smale ${ }^{1,2,4}$

\begin{abstract}
${ }^{1}$ Howard Hughes Medical Institute, Department of Microbiology, Immunology, and Molecular Genetics, and ${ }^{2}$ Molecular Biology Institute, University of California, Los Angeles, California 90095-1662 USA; ${ }^{3}$ Lymphocyte Development Group, MRC Clinical Sciences Centre, Imperial College School of Medicine, Hammersmith Hospital, London W12ONN, UK
\end{abstract}

\begin{abstract}
Ikaros is a sequence-specific DNA-binding protein that is essential for lymphocyte development. Little is known about the molecular function of Ikaros, although recent results have led to the hypothesis that it recruits genes destined for heritable inactivation to foci containing pericentromeric heterochromatin. To gain further insight into the functions of Ikaros, we have examined the mechanism by which it is targeted to centromeric foci. Efficient targeting of Ikaros was observed upon ectopic expression in 3T3 fibroblasts, demonstrating that lymphocyte-specific proteins and a lymphoid nuclear architecture are not required. Pericentromeric targeting did not result from an interaction with the Mi-2 remodeling factor, as only a small percentage of Mi-2 localized to centromeric foci in 3T3 cells. Rather, targeting was dependent on the amino-terminal DNA-binding zinc finger domain and carboxy-terminal dimerization domain of Ikaros. The carboxy-terminal domain was required only for homodimerization, as targeting was restored when this domain was replaced with a leucine zipper. Surprisingly, a detailed substitution mutant analysis of the amino-terminal domain revealed a close correlation between DNA-binding and pericentromeric targeting. These results show that DNA binding is essential for the pericentromeric localization of Ikaros, perhaps consistent with the presence of Ikaros binding sites within centromeric DNA repeats. Models for the function of Ikaros that are consistent with this targeting mechanism are discussed.
\end{abstract}

[Key words: Ikaros, TdT, lymphocytes, transcription, centromeres]

Received May 1, 2000; revised version accepted June 30, 2000.

Much of our knowledge of the events that regulate the development of mature lymphocytes from multipotent stem cell progenitors has emerged from studies of sequence-specific DNA-binding proteins that control the expression of lymphocyte-specific genes (Glimcher and Singh 1999). Ikaros is a prominent example of a DNAbinding protein that regulates multiple steps during both $\mathrm{B}$ and T lymphocyte development (Cortes et al. 1999; Cobb and Smale 2000). However, unlike most DNAbinding proteins that regulate lymphopoiesis, Ikaros may not be a typical transcription factor.

The suggestion that Ikaros performs unique functions originated in part from the observation that it is localized to foci of pericentromeric heterochromatin in some immature and mature lymphocytes (Brown et al. 1997, 1999). The pericentromeric localization of Ikaros may be conserved through evolution, as localization to phenotypically similar foci was observed in trout and man (Hansen et al. 1997; Sun et al. 1999). Interestingly, immunoFISH studies have revealed that Ikaros colocalizes

${ }^{4}$ Corresponding author.

E-MAIL steves@hhmi.ucla.edu; FAX (310) 206-8623.

Article and publication are at www.genesdev.org/cgi/doi/10.1101/ gad.816400. to centromeric foci with inactivate, developmentallyregulated genes, suggesting that Ikaros may contribute to the pericentromeric repositioning and heritable inactivation of these genes (Brown et al. 1997, 1999). More specifically, Ikaros may bind DNA elements in genes that are destined for inactivation and help recruit them to centromeric foci, where they may then assemble into heterochromatin. This hypothesis is supported by the finding that Ikaros binding sites within the promoter for the terminal transferase (TdT) gene are required for transcriptional inactivation during thymocyte development (L.A. Trinh and S.T. Smale, unpubl.). The TdT gene is one of several genes that are repositioned to centromeric foci upon inactivation (Brown et al. 1999). Thus, the binding of Ikaros to the TdT promoter may lead to pericentromeric repositioning, although direct evidence for this function has not been obtained.

Additional support for the hypothesis that Ikaros plays a role in gene inactivation or pericentromeric targeting has emerged from the finding that Ikaros and inactive genes migrate with similar kinetics toward centromeric foci when resting B cells are stimulated to enter the cell cycle (Brown et al. 1999). Furthermore, studies by Georgopoulos and colleagues have revealed that Ikaros is generally important for maintaining signaling thresholds 
during gene activation (Avitahl et al. 1999; Winandy et al. 1999). The strength of a transduced signal that is required to activate a gene is reduced when Ikaros is absent, consistent with the hypothesis that Ikaros may contribute to the maintenance of an inactive state. Finally, Ikaros has been shown to interact with two distinct histone deacetylase complexes (Kim et al. 1999).

Consistent with the hypothesis that Ikaros performs unusual functions, full-length, overexpressed Ikaros possesses only a modest capacity for activation and repression of simple reporter genes in transient and stable transfection assays (Molnár and Georgopoulos 1994; Sun et al. 1996; Koipally et al. 1999; B.S. Cobb, unpubl.). These data do not rule out the possibility that Ikaros can function as an activator or repressor, however, as activation or repression may involve mechanisms that do not yield strong activity in a typical transfection assay. Also of note, Ikaros contains domains that, when fused to a GAL4 DNA-binding domain, can activate and repress transcription in transient transfection experiments (Sun et al. 1996; Koipally et al. 1999). The functions of these domains appear to be masked in the full-length protein (B.S. Cobb, unpubl.), but it remains possible that they are unmasked by appropriate posttranslational modifications or protein-protein interactions.

Although the results summarized above have led to the hypothesis that the function of Ikaros at centromeric foci is to recruit genes undergoing downregulation, no direct evidence for this function has been obtained. This deficiency is significant because a few observations suggest alternative functions for Ikaros at centromeric foci. In particular, Ikaros appears to colocalize with DNA replication forks and to enhance genome stability, suggesting possible functions in these processes (Avitahl et al. 1999).

To explore the possibility that Ikaros recruits inactive genes to centromeric foci, we set out to test specific pre- dictions of this hypothesis. One important prediction was the following: If Ikaros recruits genes to centromeric foci, Ikaros itself is likely to be maintained at these foci via stable protein-protein interactions, leaving the DNA-binding domain free to interact with target genes. To test this prediction, we determined the pericentromeric targeting mechanism for Ikaros. Surprisingly, the results revealed that targeting requires direct DNA-binding. This targeting mechanism is incompatible with the simple hypothesis that the DNA-binding domain is dedicated to interactions with primary target genes. However, from these data, three alternative models for the function of Ikaros at pericentromeric heterochromatin can be proposed.

\section{Results}

Localization of Ikaros to pericentromeric heterochromatin following ectopic expression in 3 T3 fibroblasts

To study the mechanism of pericentromeric targeting, we first determined whether targeting is restricted to lymphocytes that naturally express Ikaros or if it can occur in other murine cell types. In other words, are other lymphocyte-specific proteins or a lymphoid nuclear architecture required for targeting? To answer this question, the localization of Ikaros was examined following ectopic expression in NIH $3 \mathrm{~T} 3$ fibroblasts, using a retroviral expression vector for the largest Ikaros isoform, isoform VI (Hahm et al. 1994).

Figure 1A shows the results of an immunoFISH experiment examining the distribution of Ikaros relative to gamma satellite DNA, an abundant repetitive element found at the centromeres of all murine chromosomes (Vissel and Choo 1989). The left panel shows the distribution of Ikaros, which was detected with an Ikaros antibody followed by an FITC-labeled secondary antibody (green). The central panel shows in the same cell the

A
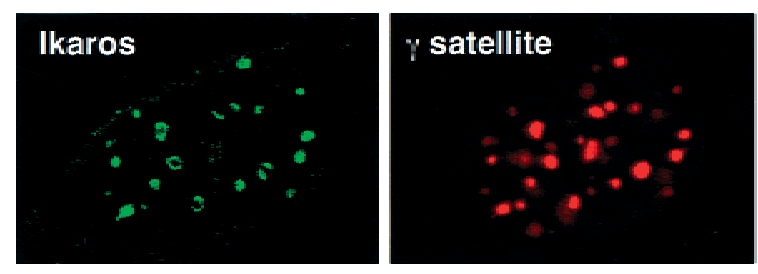

B
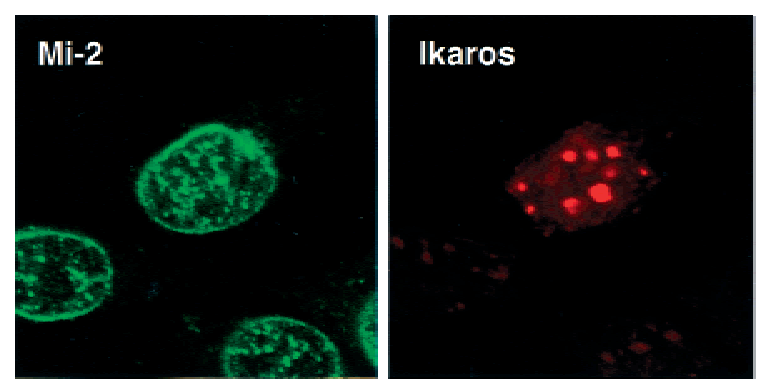

Figure 1. Targeting of Ikaros, but not Mi2 , to centromeric foci in NIH 3T3 cells. $(A)$ 3T3 cells were transduced with a retrovirus engineered to express Ikaros isoform VI. Cells were stained with an Ikaros antibody (green, first panel) and with a FISH probe for gamma satellite DNA (red, second panel). The third panel shows the combined images. $(B)$ NIH3T3 cells expressing Ikaros isoform VI were costained for Ikaros and Mi-2. Ikaros was detected with the monoclonal Ikaros antibody 2A9 followed by a Texas red-labeled goat antimouse IgG $\mathrm{F}(\mathrm{c})$ antibody (red, second panel). Mi-2 was detected with a rabbit polyclonal antibody against $\mathrm{Mi}-2$ (provided by Weidong Wang, NIH) followed by an FITC-labeled goat anti-rabbit antibody (green, first panel). The third panel shows the combined images. 
distribution of gamma satellite DNA, which was detected by FISH using a fluoro-RED-labeled DNA probe (red). The right panel shows the combined images. Ikaros appears to surround the gamma satellite DNA, which is reminiscent of the results obtained in cycling lymphocytes and cell lines (Brown et al. 1997). A similar distribution pattern of Ikaros was observed following retroviral transduction of other murine lines, including hepatoma (Hepa 1), muscle (C2C12), and neuronal (W20) lines (data not shown). Therefore, localization of Ikaros to pericentromeric heterochromatin does not require lymphocyte-specific factors or a lymphoid nuclear architecture.

The Mi-2 nucleosome remodeling factor does not recruit Ikaros to centromeric foci

Ikaros was recently found to associate with the NURD chromatin remodeling/histone deacetylase complex through a direct interaction with the Mi-2 (or CHD4) subunit (Kim et al. 1999). Because Mi-2 and Ikaros colocalized to regions of pericentromeric heterochromatin in some $\mathrm{T}$ cells, and because $\mathrm{Mi}-2$ is ubiquitously expressed, the NURD complex might be responsible for the pericentromeric recruitment of Ikaros in $3 \mathrm{~T} 3$ fibroblasts. To test this possibility, the relative subcellular distributions of Mi-2 and Ikaros were examined in Ikaros-expressing 3T3 cells. The confocal images in Figure 1B show the distribution pattern of each protein. Mi-2 was detected with a rabbit polyclonal antibody and an FITClabeled secondary antibody (green). Ikaros was detected with a mouse monoclonal antibody and a Texas red-labeled secondary antibody (red). Very little, if any, Mi-2 protein colocalized with Ikaros, as Mi-2 was concentrated at the nuclear periphery and at numerous other foci that were distinct from those containing Ikaros. Consistent with the confocal data, quantitative coimmunoprecipitation experiments from primary splenocyte extracts have shown that only a small fraction of the Mi-2 protein within a cell population is stably associated with Ikaros and only about $20 \%$ of Ikaros is associated with Mi-2 (data not shown). The detection of an Ikaros-Mi-2 interaction is consistent with the results described in Kim et al. (1999). However, this interaction does not appear to be responsible for the pericentromeric targeting of Ikaros.

Amino- and carboxy-terminal zinc finger domains are required for pericentromeric targeting

To examine in greater depth the mechanism of Ikaros targeting to pericentromeric heterochromatin, a series of Ikaros deletion mutants was analyzed to identify the targeting domains (Fig. 2A, top). The starting point for this analysis was the largest of the Ikaros isoforms (isoform VI), which contains four classic $\mathrm{C}_{2} \mathrm{H}_{2}$ zinc fingers near the amino terminus and two additional $\mathrm{C}_{2} \mathrm{H}_{2}$ zinc fingers at the carboxyl terminus (Hahm et al. 1994). The amino-terminal zinc fingers are responsible for sequence-specific DNA-binding (Hahm et al. 1994; Molnár and Georgopoulos 1994), with the carboxy-terminal fingers serving as a dimerization domain (Sun et al. 1996; Hahm et al. 1998). Each mutant protein, containing an amino-terminal HA epitope tag, was expressed from a retroviral vector by transduction of 3T3 fibroblasts. These cells were chosen for the analysis instead of lymphocytes because they appear to lack endogenous Ikaros family members, which in lymphocytes would form dimers with the exogenous mutant proteins and most likely influence their localization. Given that pericentromeric targeting is highly unusual among sequencespecific DNA-binding proteins, 3T3 cells should be suitable for this analysis because it is difficult to imagine targeting in fibroblasts via a different mechanism than that used in lymphocytes. As in all experiments described below, Ikaros localization was determined by confocal microscopy, using Ikaros polyclonal antibodies directed against either an amino-terminal or a carboxyterminal domain and an FITC-labeled secondary antibody (green). Nuclear DNA was stained with propidium iodide (red).

Representative confocal images of the transduced 3T3 cells are shown in Fig. 2A (bottom). As expected, no Ikaros staining was observed in cells infected with a retrovirus lacking an Ikaros cDNA (panel 3T3). In contrast, full-length Ikaros isoform VI (panel VI) exhibited the punctate staining pattern characteristic of pericentromeric localization (Fig. 1A). A similar staining pattern was observed with Ikaros isoform V (Fig. 2A, panel V), a naturally occurring splice variant. This isoform lacks the region encoded by exon 3 , and thus, the first zinc finger. The similar localization of isoforms V and VI is noteworthy because they are the two predominant isoforms expressed in lymphocytes (Hahm et al. 1994; Molnár and Georgopoulos 1994).

In addition to isoforms V and VI, four other deletion mutants localized predominantly to the nucleus and exhibited the punctate staining pattern characteristic of pericentromeric targeting (panels 54-C, d235-362, d363451 , and dF1). In contrast, a mutant that lacks the carboxy-terminal zinc finger dimerization domain (panel N-458) was distributed heterogeneously within the nucleus, although it was excluded from large patches that are likely to be nucleoli. This distribution suggests an absence of pericentromeric targeting. Finally, four proteins exhibited partial or complete localization to the cytoplasm: I (corresponding to Ikaros isoform I), dF2, $\mathrm{dF} 3$, and dF4. All of these proteins lack fingers 2, 3, or 4, demonstrating that nuclear localization is largely determined by this region, even though it lacks a classic nuclear localization signal. Interestingly, although only a fraction of the $\mathrm{dF} 4$ protein was found in the nucleus, this fraction exhibited the characteristic punctate staining pattern.

Figure 2A summarizes the above results. The deletion mutants that retain pericentromeric targeting are shown in blue, and those found solely in the cytoplasm or broadly distributed throughout the nucleus are shown in 
A
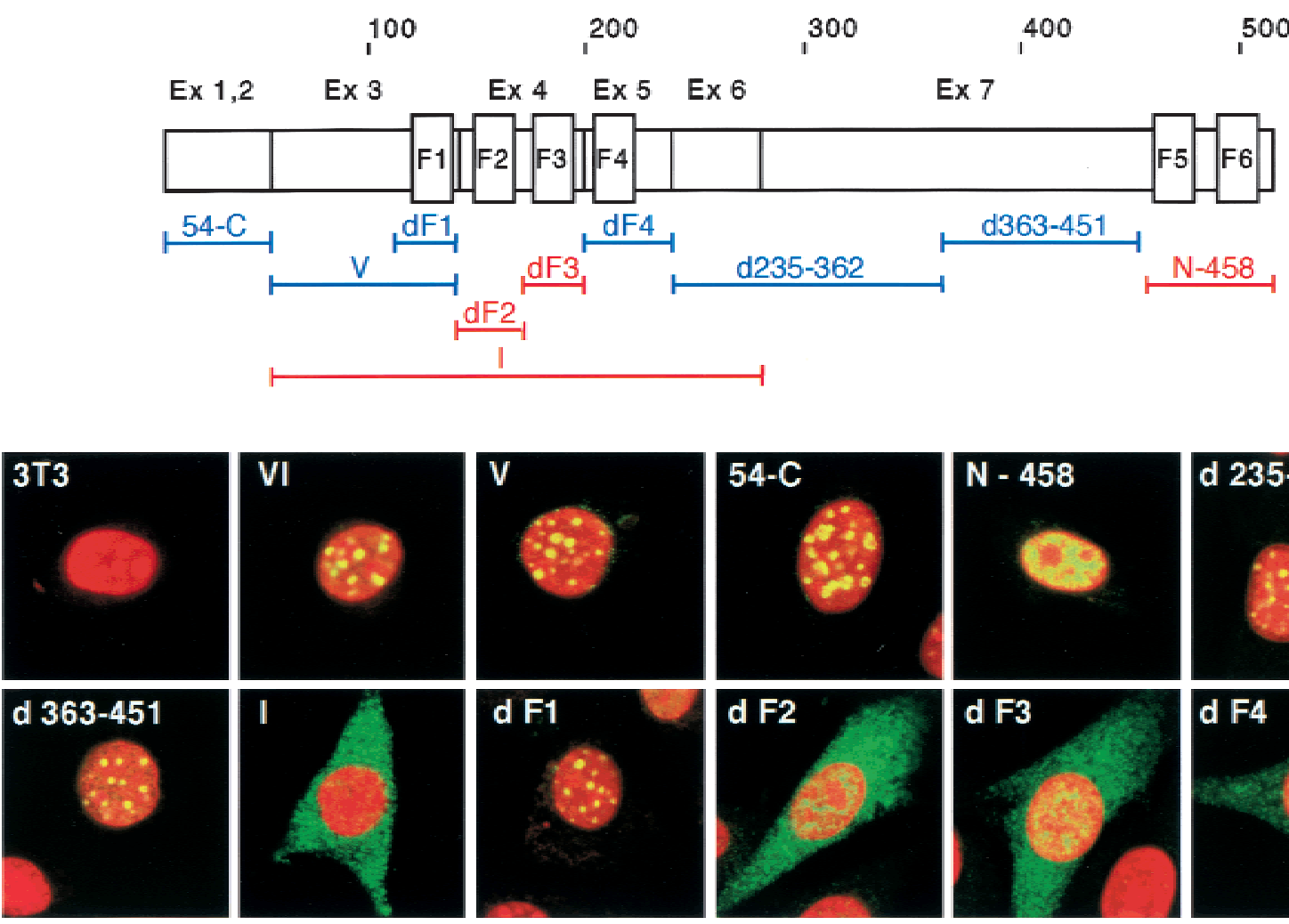

B

NLS
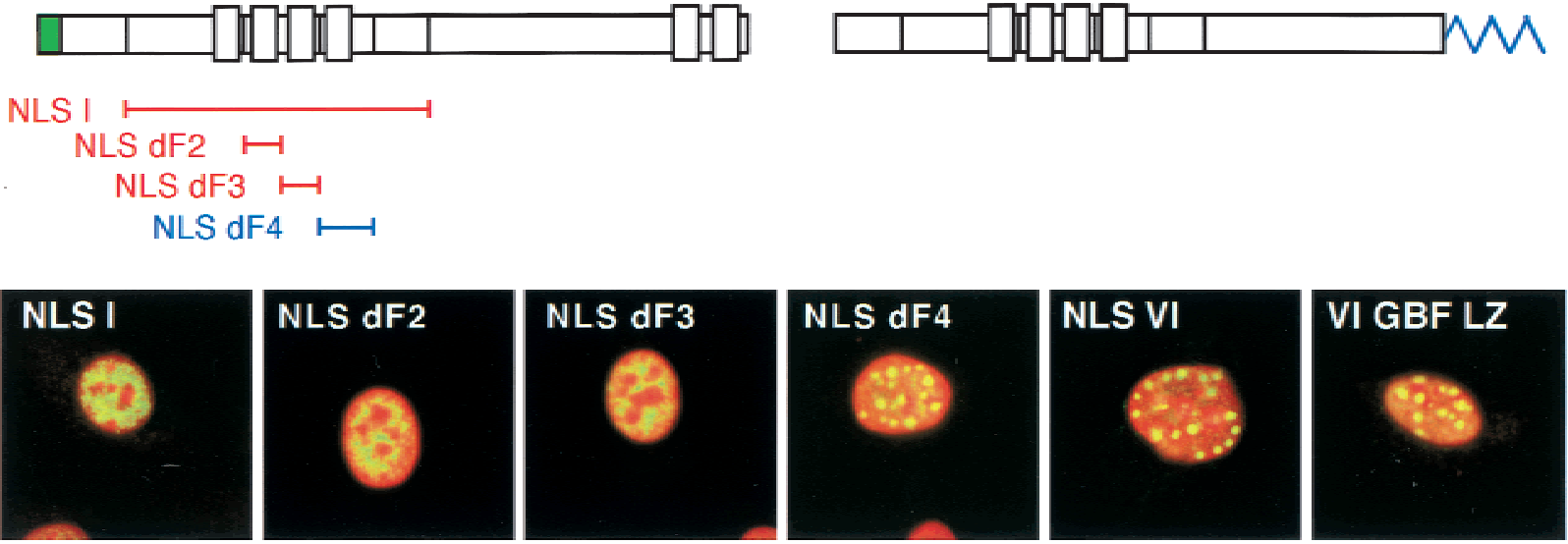

Figure 2. Identification of Ikaros domains involved in pericentromeric targeting. (A) A diagram of Ikaros isoform VI is shown at the top. The amino acid number and the regions encoded by the seven exons are indicated above the diagram, with exon boundaries indicated by vertical lines. The six zinc fingers are depicted as boxes. The first four are involved in DNA binding, and the last two, in dimerization. Below the diagram, the horizontal lines indicate the regions deleted in each mutant. Those in red disrupted pericentromeric targeting, and those in blue retained targeting. The confocal images of 3T3 cells transduced with retroviruses are shown at the bottom. For the first image (3T3), cells were infected with a retrovirus without an Ikaros cDNA. In all images, cells were stained with an Ikaros antibody (green) and with propidium iodide (red) to visualize DNA. (B) The diagrams at the top represent the constructs used for this analysis. To determine whether the presence of a nuclear localization signal (NLS) can overcome the requirement for the amino-terminal zinc fingers, an SV40 T antigen NLS was positioned at the amino terminus of Ikaros (left diagram, green box). The regions deleted in each mutant are indicated by horizontal lines, with the mutants retaining and losing pericentromeric targeting indicated in blue and red, respectively. To determine whether a heterologous dimerization domain can overcome the requirement for the carboxy-terminal zinc fingers, these fingers were replaced by a leucine zipper from the Arabidopsis thaliana GBF protein (right diagram, indicated by the kinked blue line). The confocal images are shown at the bottom and were prepared as described in $A$.

red. Taken together, the results indicate that amino-terminal zinc fingers 2 and 3 and carboxy-terminal fingers 5 and 6 are the only regions of Ikaros that are essential for pericentromeric targeting. 


\section{Analysis of the two essential zinc finger domains}

Because the deletion analysis indicated that only the amino- and carboxy-terminal zinc fingers are required for pericentromeric targeting, additional experiments were performed to elucidate the essential features of these domains. For example, the amino-terminal DNA-binding zinc fingers might be required for the nuclear localization of Ikaros, with the carboxy-terminal fingers responsible for pericentromeric targeting within the nucleus. Conversely, the carboxy-terminal zinc fingers might be required only for dimerization, with the DNA-binding fingers responsible for pericentromeric targeting of Ikaros dimers.

To determine whether the DNA-binding zinc fingers are required for pericentromeric targeting or merely for nuclear localization, retroviral expression constructs were prepared for variants of mutants $I, d F 2, d F 3, d F 4$, and wild-type Ikaros isoform VI, with each variant containing a nuclear localization sequence (NLS) from the SV40 large T antigen. The SV40 NLS facilitated efficient nuclear localization of all four mutant proteins and of the wild-type control protein (Fig. 2B, first five panels). However, only wild-type isoform VI and one mutant protein, NLS dF4, exhibited pericentromeric targeting. The other three mutants were distributed heterogeneously within the nucleus. These results show that the nuclear localization imparted by zinc fingers 2 and 3 is insufficient for pericentromeric targeting; rather, these fingers must contribute another function that is essential for targeting (see below).

To determine whether the carboxy-terminal zinc fingers are directly involved in targeting or are merely required for dimerization, a retroviral expression construct was prepared for a variant of Ikaros isoform VI in which the carboxy-terminal fingers were replaced with a leucine zipper domain from an Arabidopsis thaliana protein, GBF1 (Schindler et al. 1992). The expectation was that the leucine zipper would support dimerization, but would lack other properties of the carboxy-terminal zinc fingers that may be required for pericentromeric targeting. Interestingly, this protein (VI GBF LZ) exhibited the punctate staining pattern characteristic of pericentromeric localization (Fig. 2B, last panel). Thus, the carboxy-terminal zinc fingers appear to be required for pericentromeric targeting only because dimerization is essential for targeting.

To confirm that the VI GBF LZ protein was capable of dimerization, coimmunoprecipitation experiments were performed. An antibody against an HA epitope tag, which was fused to the VI GBF LZ protein, was capable of precipitating VI GBF LZ as well as a co-expressed, untagged variant of Ikaros isoform $\mathrm{V}$ containing the carboxy-terminal leucine zipper (data not shown). It is interesting to note, however, that coimmunoprecipitation mediated by the leucine zipper was less efficient than that observed with the native carboxy-terminal zinc fingers of Ikaros (data not shown), suggesting a reduced dimerization affinity. Nevertheless, this reduced affinity appears to be sufficient for targeting to pericentromeric heterochromatin.
Localization to pericentromeric heterochromatin correlates with high-affinity DNA binding

The requirement for the DNA-binding zinc fingers raises the intriguing possibility that pericentromeric targeting results from the direct binding of Ikaros to DNA elements that are present at centromeric foci. This hypothesis is consistent with the data described above. However, targeting by direct DNA binding was unexpected because the current model proposes that the Ikaros DNA-binding domain interacts with control regions in lymphocyte-specific target genes, thereby recruiting those genes to centromeric foci (Brown et al. 1997, 1999). A scenario that appeared to be more consistent with this model was that Ikaros would be positioned at centromeric foci through protein-protein interactions. Because zinc fingers 2 and 3 may be capable of mediating both protein-protein and protein-DNA interactions, a critical goal was to determine which biochemical activity of these fingers determines pericentromeric targeting. As a first step toward this goal, the DNA-binding properties of the mutant proteins described above were examined to determine if a correlation exists between DNA binding and pericentromeric targeting.

Nuclear extracts were prepared from 293T cells transfected with expression plasmids for each of the HAtagged wild-type and mutant proteins. A Western blot was then performed with antibodies against the HA epitope. For this experiment and the subsequent gel shift experiments, extract concentrations were adjusted to yield similar amounts of each protein by diluting extracts containing larger amounts of Ikaros protein with extracts from vector-transfected cells. The Western blot results (Fig. 3A, top panel) revealed that all mutant proteins were present at similar concentrations within the normalized extracts.

Gel shift experiments were performed with four different probes containing Ikaros recognition sites (Fig. 3A, panels 2-5). An analysis of multiple probes was necessary to provide a fair assessment of the binding properties of the mutant proteins, because different results were obtained with each probe. The first probe, IK bs4, contains a high-affinity Ikaros binding site derived from binding site selection studies (Fig. 3A, panel 2; see Fig. 3B for probe sequences) (Molnár and Georgopoulos 1994). With this probe, the proteins targeted to centromeric foci (indicated in blue in Fig. 3A) formed the most abundant gel shift complexes; proteins that did not localize to centromeric foci (indicated in red) formed no detectable complex or a low-abundance complex. The proteins that formed no detectable complex were those containing deletions of fingers 2 and 3 (dF2, dF3, NLS $\mathrm{dF} 2$, and NLS $\mathrm{dF} 3$ ), demonstrating that these two fingers, but not fingers 1 and 4, are essential for binding to the IK Bs4 probe. The mutant that formed a low-abundance complex lacked the carboxy-terminal zinc fingers (N-458), suggesting that dimerization is required for high-affinity binding. These results establish an initial correlation between DNA binding and pericentromeric targeting. 
A

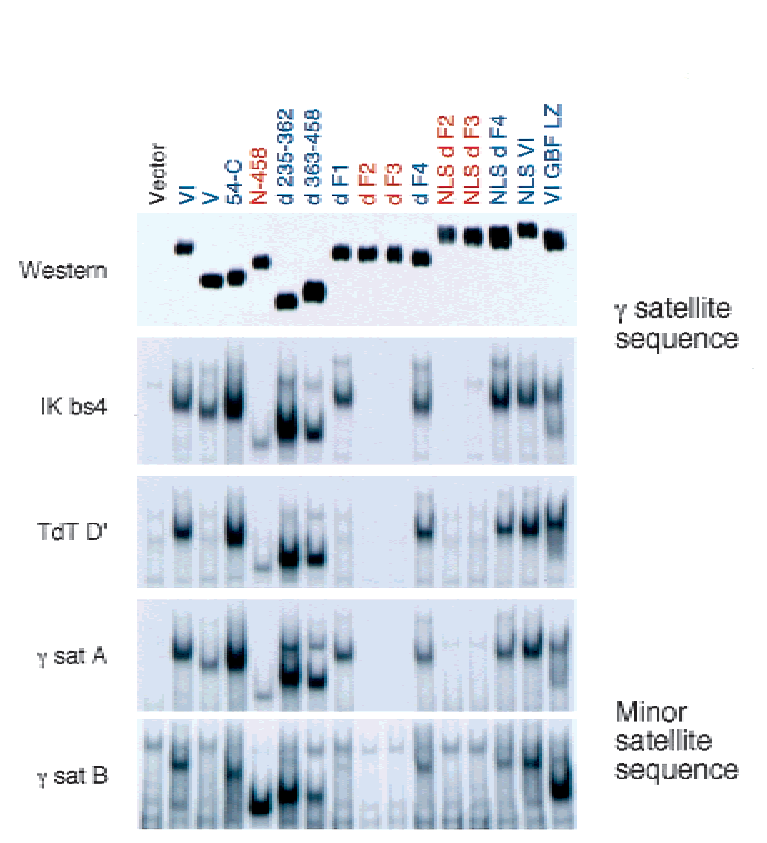

B
Hs 2 mer bs ATCCATAGGGAAARTGITTTCCCIGACATC

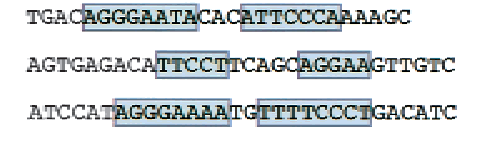

$\eta$ sat $\mathrm{A}$

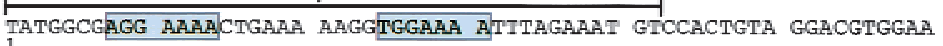

1 TATGGCAAGA TGACGAAATC ACTAAAAAAC GTGAAAAATG AGAAATGCAC ACTGAAGGAC CTGGAATATG

횬ㄴ

GCGAGAAAAC TGAAAATCAC GGAAAATGAG AAATACACAC TTTAGGACGT GAAA

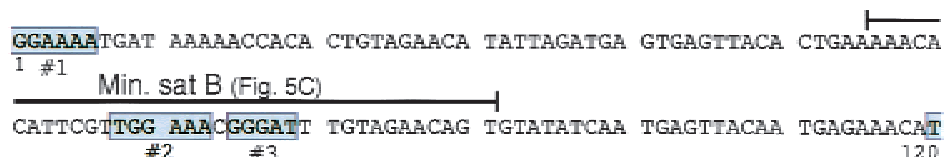

Figure 3. DNA binding of the mutant Ikaros proteins. (A) 293T cells were transfected with expression vectors for the Ikaros proteins indicated at the top (vector indicates transfection with the expression vector lacking an Ikaros cDNA). The Ikaros proteins labeled in blue were localized to regions of pericentromeric heterochromatin whereas those labeled in red were not. A Western blot probed with HA antibodies shows the relative amounts of each protein in the normalized nuclear extracts (top panel). To achieve similar levels of Ikaros proteins, extracts were diluted when necessary with nuclear extract from cells transfected with the empty vector. Gel shifts were performed with these nuclear extracts using four different Ikaros binding site probes (panels 2-5). Only the portion of the gel containing the protein-DNA complex is shown. All experiments were performed with an excess of labeled probe. $(B)$ The sequences of the DNA probes used for gel shift experiments are shown, along with the entire sequence of the $\gamma$ satellite and minor satellite repeats. Motifs that resemble the Ikaros consensus are shaded. IK bs4 and Hs 2mer bs (used in Fig. 5C) contain high-affinity binding sites derived from binding site selection studies. TdT $\mathrm{D}^{\prime}$ is a naturally occurring binding site from the TdT promoter. Within the satellite sequences, the probe sequences analyzed are overlined.

The gel shift results obtained with the second radiolabeled probe (TdT D'; Fig. 3A, panel 3) supported this hypothesis, although the correlation was imperfect. This probe contains the two naturally occurring Ikaros binding sites within the $\mathrm{D}^{\prime}$ element of the TdT promoter (Hahm et al. 1994). The binding profile obtained was similar to that obtained with IK bs4, but complex formation was disrupted when finger 1 was removed (panel 3, $\mathrm{V}$ and $\mathrm{dF} 1$ ). Fingers 1,2 , and 3 are therefore required for efficient binding to this element.

The third and fourth probes contain potential Ikaros binding sites from the gamma satellite sequences that are abundant at the centromeres of all murine chromosomes. These sites were chosen because the above results raise the possibility that pericentromeric targeting requires direct DNA binding to centromeric sequences. On the basis of the Ikaros consensus sequences defined by binding site selection (Molnár and Georgopoulos 1994; Hahm et al. 1998), five potential recognition sites were identified within the gamma satellite sequence (Fig. 3B) (Vissel and Choo 1989). Three potential sites were found within the minor satellite sequence (Wong and Rattner 1988), which is also abundant at murine centromeres. The two probes used to analyze the mutant proteins, $\gamma$ sat $A$, containing gamma satellite binding sites 1 and 2 (Fig. 3B) and $\gamma$ sat B, containing gamma satellite binding site 3 (Fig. 3B), exhibited relatively highaffinity interactions with Ikaros when compared to probes containing the other potential binding sites (data not shown).

The binding profile observed with the $\gamma$ sat A probe (Fig. 3A, panel 4) was similar to the IK bs4 profile. The profile observed with the $\gamma$ sat B probe (panel 5) was similar to the TdT D' profile, with one important exception: Monomeric proteins appeared to yield more abundant complexes than dimeric proteins. This monomer preference was apparent with mutant N-458 (lacking the carboxy-terminal zinc fingers), which formed a more abundant complex than the proteins that retain the carboxy-terminal fingers. Furthermore, the predominant complex formed by the VI GBF LZ protein on this probe migrated more rapidly than the predominant complex formed by the same protein on the other three probes. A reasonable explanation for this result is that the faster and slower migrating complexes represent monomers and dimers, respectively.

The fact that Ikaros dimers do not preferentially bind the $\gamma$ sat B probe is consistent with the presence of only 
one Ikaros recognition site on this probe, which eliminates the possibility of cooperative binding by the two subunits of an Ikaros dimer. However, it is not clear why monomeric proteins actually form more abundant protein-DNA complexes with this probe than dimeric proteins when similar amounts of monomeric and dimeric protein are analyzed. One possibility is that the monomeric proteins expressed in $293 \mathrm{~T}$ cells possess a higher specific activity with respect to DNA-binding, perhaps because dimeric proteins are more likely to form aggregates. Alternatively, Ikaros may undergo a conformational change upon dimerization that reduces its affinity for some DNA sequences.

Taken together, the results in Figures 2 and 3 reveal a close correlation between pericentromeric targeting and binding to the IK bs 4 and $\gamma$ sat A probes. An imperfect correlation was observed between pericentromeric targeting and binding to the TdT $\mathrm{D}^{\prime}$ and $\gamma$ sat $\mathrm{B}$ probes.

\section{Analysis of substitution mutants that alter zinc fingers 2 and 3}

The finding that zinc fingers 2 and 3 are essential for both pericentromeric targeting and DNA binding supports the hypothesis that DNA binding is directly responsible for targeting. However, these data do not rule out the alternative possibility that a protein-protein interaction surface within fingers 2 and 3 is responsible for targeting. To examine the correlation between DNA binding and pericentromeric targeting in greater detail, substitution mutants were analyzed that replace amino acids within fingers 2 and 3 with amino acids from another zinc finger. If fingers 2 and 3 mediate protein-protein interactions that are critical for pericentromeric targeting, one would expect to find mutations that disrupt targeting but not DNA binding, and vice versa.

First, fingers 2 and 3 were individually replaced with Ikaros zinc finger 5, yielding mutants F2 $>5$ and F3 $>5$, respectively (Fig. 4A). Finger 5 was chosen because, with the exception of the critical cysteines and histidines, its sequence is almost completely divergent from that of fingers 2 and 3 . Both mutant proteins were primarily cytoplasmic (Fig. 4B) and neither protein bound DNA (Fig. 4C), similar to the properties of proteins containing deletions of finger 2 or 3 (see Fig. 2).

Next, 16 substitution mutants were analyzed in which 3-8-amino acid clusters within fingers 2 or 3 were replaced by the corresponding amino acids from finger 5 (Fig. 4A). Only four of these mutants (F2 > 5 145-52 and 164-66; F3 > 5 173-77 and 188-90) exhibited the punctate staining pattern characteristic of pericentromeric targeting (Fig. 4B). Importantly, these were the same four mutants that formed abundant gel shift complexes with at least three of the four DNA probes (Fig. 4C). As shown in Figure 4B, the other 12 mutants in this series were either (1) entirely nuclear with no evidence of pericentromeric targeting (F2 > 5 153-56; F3 > 5 173-80, 188-95, 179-81, and 185-187); (2) partially nuclear with no evidence of pericentromeric targeting (F2 > 5 160-67, 157-
59, and160-162; F3 > 5 192-94); or (3) entirely cytoplasmic (F2 > 5 153-59; F3 > 5 181-87 and 182-84). Importantly, among these 12 non-centromeric mutants, only two formed detectable gel shift complexes (Fig. 4C): F3 > 5 173-80 formed a weak complex with the $\gamma$ sat A probe and F3 > 5 192-94 formed weak complexes with three of the four probes.

Taken together, the results in Figure 4 localized the zinc finger region required for both pericentromeric targeting and high-affinity DNA binding to three clusters of amino acids: a ten-residue segment of finger 2 (amino acids 153-162), a nine-residue segment of finger 3 (amino acids 179-187), and a three-residue segment of finger 3 (amino acids 192-194).

\section{Analysis of single amino acid substitutions}

The above results revealed a close correlation between DNA binding and pericentromeric targeting, providing strong support for the hypothesis that direct DNA binding, and not a protein-protein interaction, is responsible for targeting. However, the results remain inconclusive because the amino acid regions that are critical for targeting are large enough to include both protein-DNA and protein-protein interaction surfaces. Some mutants could also disrupt the overall structure of the zinc fingers, thereby disrupting all of their functions. Given the unexpected nature of the results, combined with the importance of understanding the pericentromeric targeting mechanism, a more rigorous test of the hypothesis was required. To this end, single amino acid substitutions within the three critical regions of fingers 2 and 3 were analyzed. To minimize the possibility of disrupting the zinc finger structure in this mutant series, an alanine was introduced at each position, or when the wild-type amino acid was an alanine, a leucine was introduced.

Overall, 26 substitution mutants were analyzed (Fig. $5 \mathrm{~A})$, including 22 mutants that alter the amino acids within the critical regions (amino acids 153-162, 179187, and 192-194), and four mutants that alter other amino acids (amino acids 174 and 188-190). Only ten of the 26 mutants disrupted pericentromeric targeting (indicated in red in Fig. 5A). Four of the ten disruptive mutants were at least partially cytoplasmic (154 F > A, 157 $\mathrm{K}>\mathrm{A}, 162 \mathrm{R}>\mathrm{A}, 184 \mathrm{R}>\mathrm{A}$ ), revealing that they are the only amino acids that are essential for nuclear localization.

It is interesting to note that, within each region encompassed by a disruptive, triple amino acid mutant in Figure 4, there was generally at least one disruptive single amino acid mutant. The two exceptions were mutants F3 > 5 185-87 and F3 > 5 192-94, as none of the single amino acid substitutions in these regions disrupted pericentromeric targeting. Perhaps, the introduction of amino acids from finger 5 disrupted the overall zinc finger structure, whereas the alanines and leucines used in the single amino acid series were not disruptive. It is noteworthy that the single mutant $188 \mathrm{~L}>\mathrm{A}$ disrupted pericentromeric targeting, whereas the triple mu- 
A

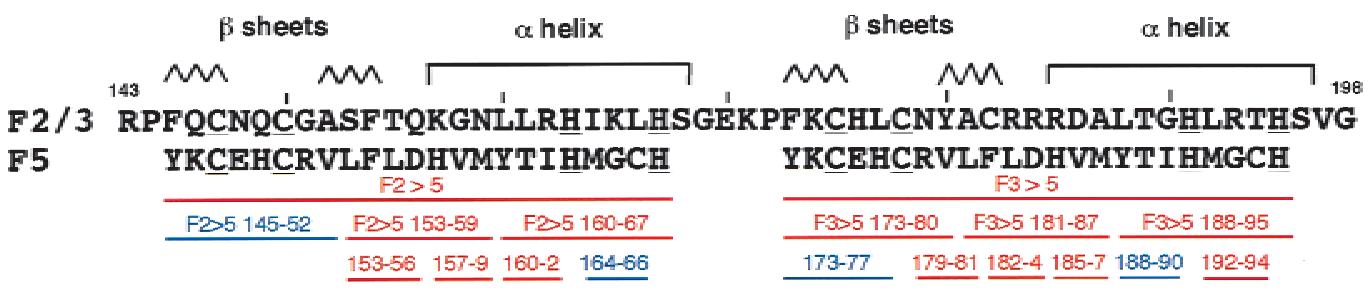

B
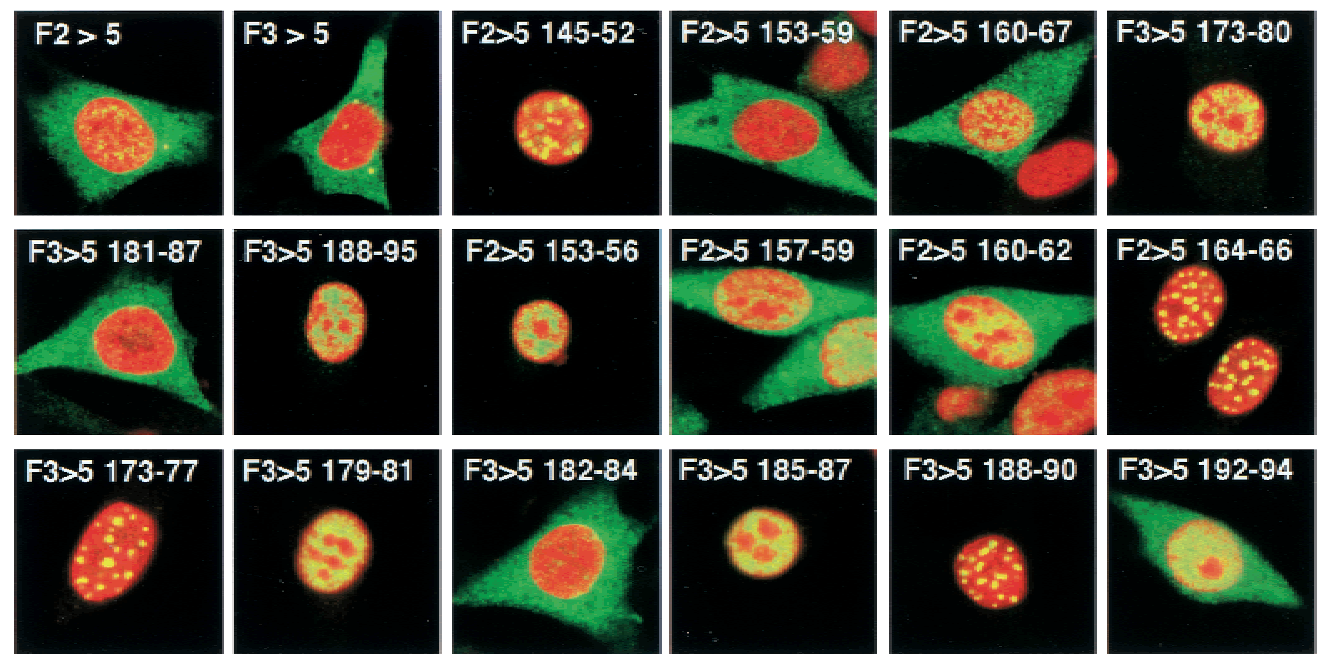

C

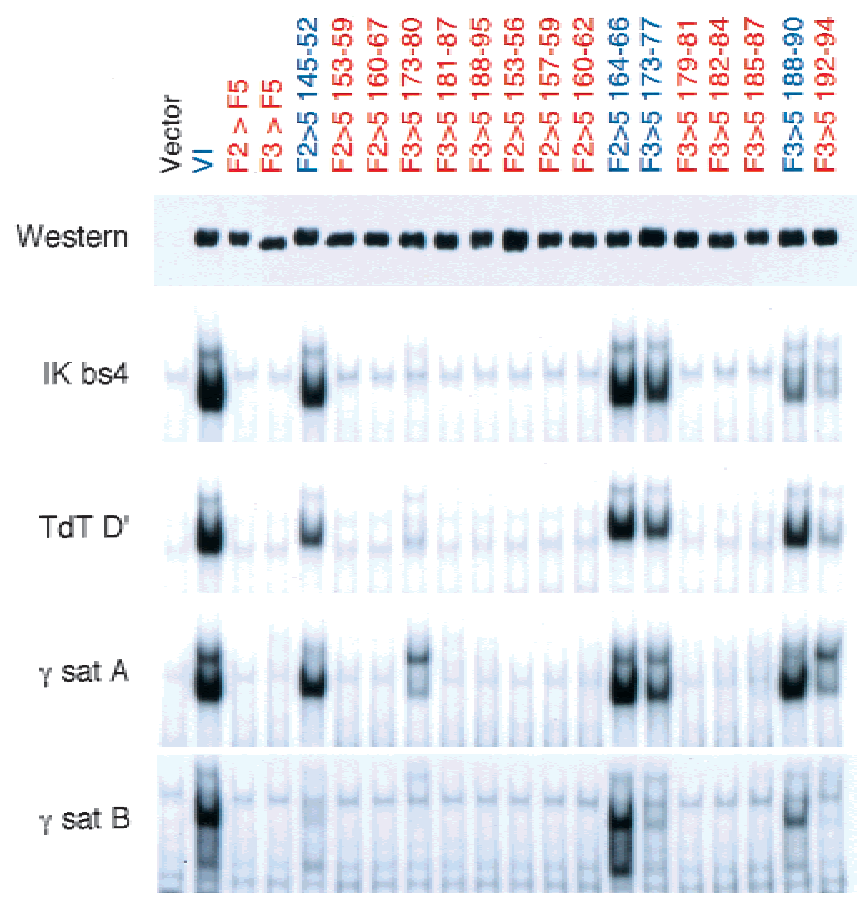

Figure 4. Substitution mutant analysis of fingers 2 and 3. (A) The amino acid sequences of fingers 2 and 3 (residues 143-198 of Ikaros isoform VI) are shown. The positions of the $\beta$ sheets and $\alpha$ helixes found in all known zinc finger structures are shown at the top. Vertical lines are placed above every tenth residue, and the critical cysteines and histidines are underlined. Below both fingers, the sequence of Ikaros finger 5 is shown for comparison. The horizontal lines at the bottom indicate the regions in finger 2 or 3 that were substituted with the corresponding regions of finger 5 . The numbers indicate the amino acids substituted in each mutant. Ikaros mutants shown in blue localized to regions of pericentromeric heterochromatin, and those shown in red did not. $(B)$ The confocal images, performed as described in the legend to Figure 1, are shown. $(C)$ The DNA-binding activities of the Ikaros mutants were determined as described in the legend to Figure 3. A Western blot performed with HA antibodies is shown (top panel), revealing the relative concentrations of the Ikaros proteins in the normalized extracts. Gel shift results obtained with the four different Ikaros binding site probes are shown (panels 2-5). Only the portion of the gel containing the bound protein is shown. 
Cobb et al.

A

$\beta$ sheets

$\alpha$ helix

$B$ sheets

$\alpha$ helix

${ }_{143} M, M M_{* * 1 *} M M_{* * *} \Gamma_{198}$ F2 / 3 RPFQCNQĆGASFTQKGNLLRHIKLHSGEKPFRCHLCNYACRRRDALTGHLRTHSVG AAAAAAAAAA

DNA binding - $\pm \pm \pm-\quad-$

A AALAAAAALAAA AAA

B
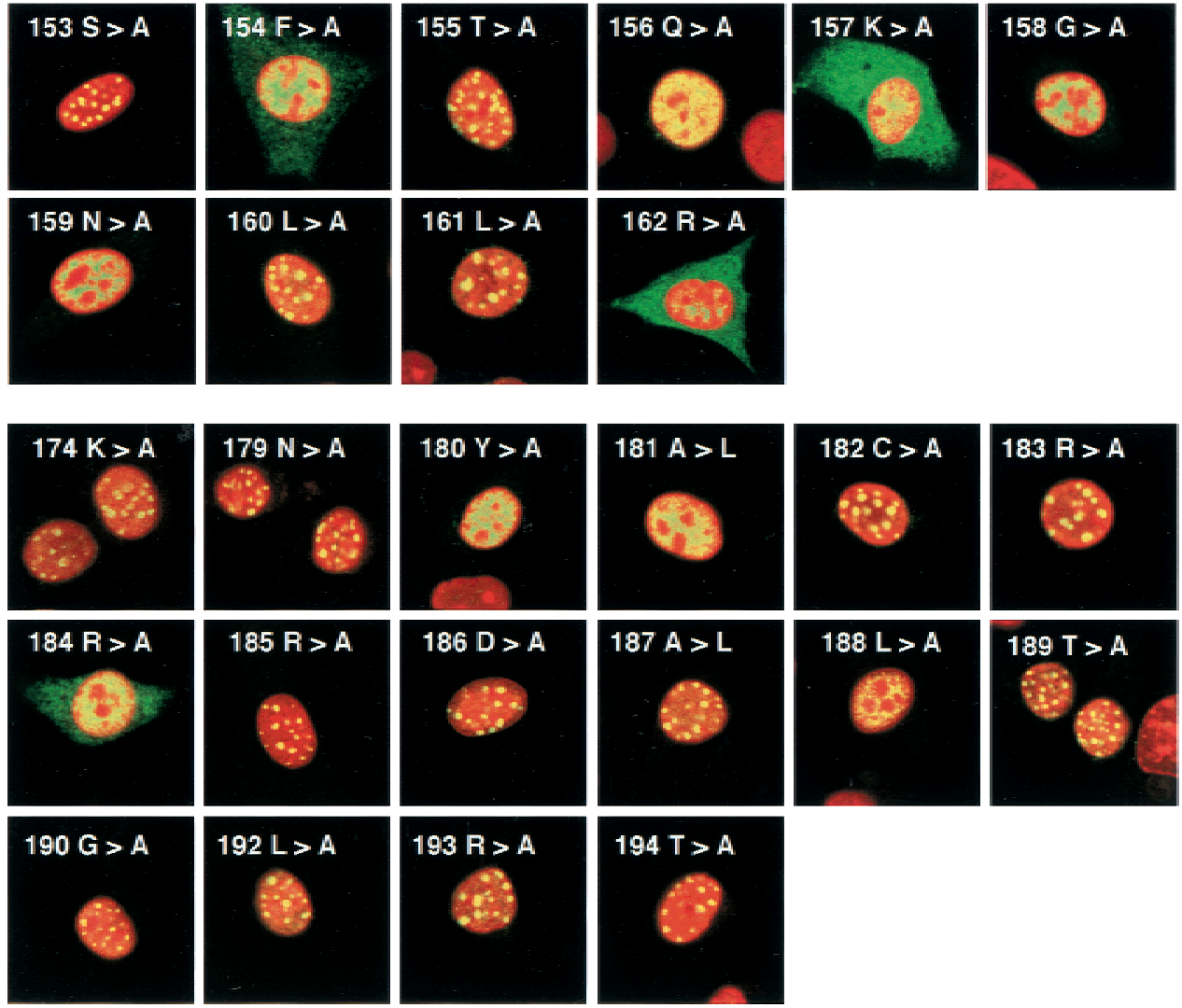

C

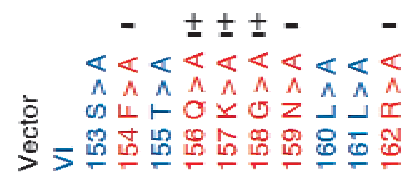

Western

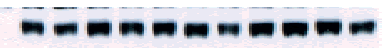

IK bs4

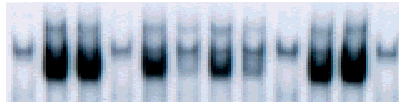

TdT D'

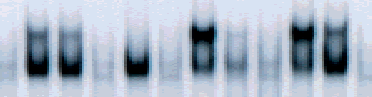

$\gamma$ sat A

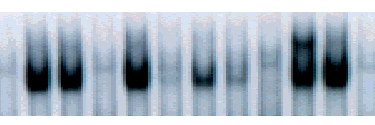

$\gamma$ sat B

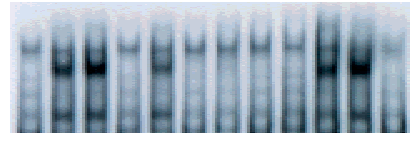

Hs 2mer bs

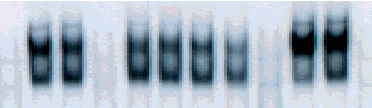

Min. sat $B$
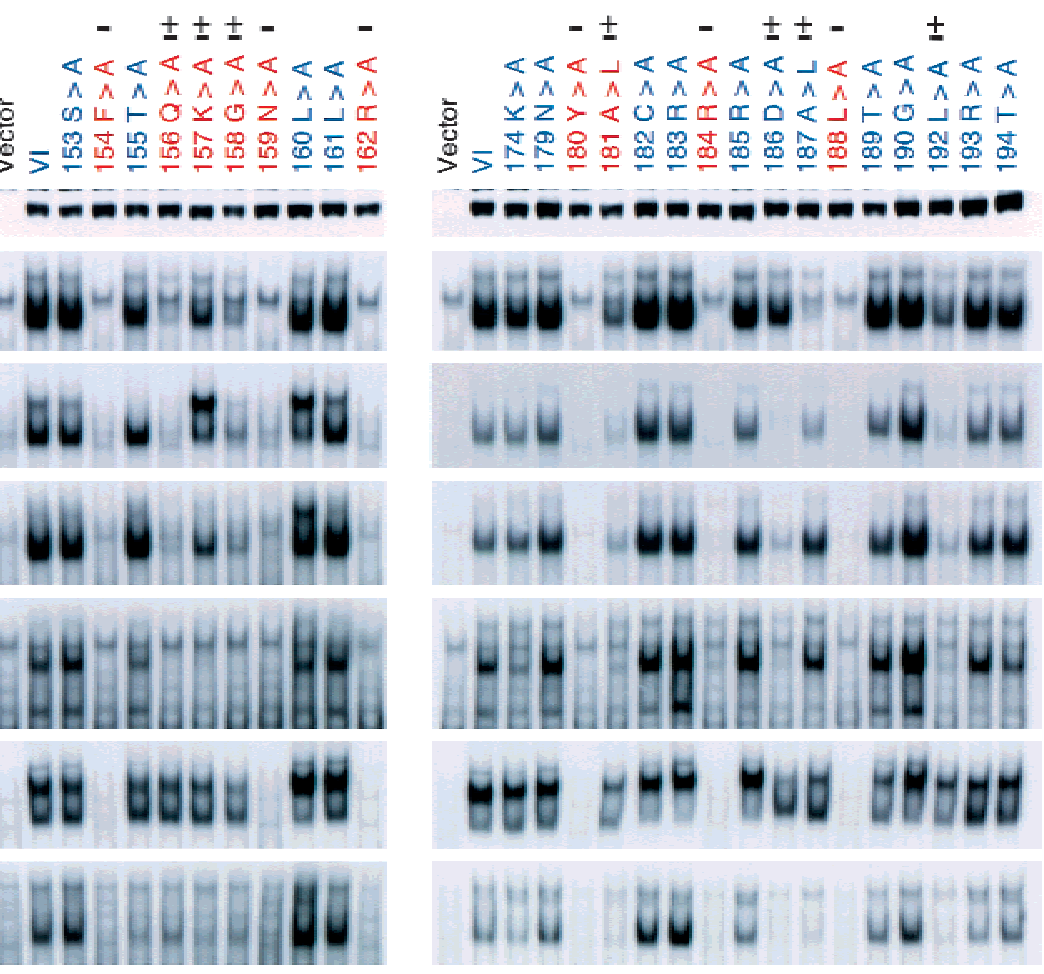
tant F3 > $5188-190$ (188 L to Y, 189 T unchanged, and 190 G to I) was not disruptive. One possibility is that the larger hydrophobic side-chains found on leucine and tyrosine are tolerated at position 188, whereas the shorter side-chain of alanine is not tolerated.

To evaluate the correlation between DNA binding and pericentromeric targeting, the DNA-binding activities of the mutant proteins were determined. Six different probes were used for this analysis to extend our knowledge of binding site variability. The two new probes were Hs 2 mer bs, derived from the binding site selection studies of Hahm et al. (1998), and Min. sat B, containing minor satellite binding sites 2 and 3 (see Fig. 3B). An examination of the binding data (Fig. 5C) revealed that the Ikaros mutants could be placed into three general categories: (1) mutants that efficiently bound five or, in most instances, all six of the probes $(13$ mutants, indicated by the absence of a plus or minus sign at the top of Figures 5A and 5C); (2) mutants that were unable to form abundant gel shift complexes with any of the DNA probes (six mutants, indicated by a minus sign in Figure $5 \mathrm{~A}$ and at the top of Figure $5 \mathrm{C}$ ); and (3) mutants that formed abundant complexes with only one to four of the DNA probes (seven mutants, indicated by \pm in Figures $5 \mathrm{~A}$ and $5 \mathrm{C})$.

A comparison of the confocal and DNA-binding data revealed that all 13 mutants in the first category with respect to DNA binding (eg., abundant complex formation with five or six probes) exhibited the punctate staining pattern characteristic of pericentromeric targeting. In contrast, none of the six mutants in the second category with respect to DNA binding (eg., little or no binding to all of the probes) exhibited pericentromeric targeting. The seven mutants in the third category (efficiently binding to only one to four probes) yielded mixed results, with three exhibiting pericentromeric targeting (186 $\mathrm{D}>\mathrm{A}, 187 \mathrm{~A}>\mathrm{L}$, and $192 \mathrm{~L}>\mathrm{A}$ ), and the remaining four distributed throughout the nucleus (156 Q > A, 157 $\mathrm{K}>\mathrm{A}, 158 \mathrm{G}>\mathrm{A}$, and $181 \mathrm{~A}>\mathrm{L}$ ).

Overall, the data reveal a striking correlation between DNA binding and pericentromeric targeting, with all 19 mutants in the two extreme categories with respect to DNA binding (categories 1 and 3 ) consistent with the correlation. The results obtained with the seven mutants in category 2 are difficult to evaluate because of the DNA-binding variability. Nevertheless, given the relatively small number of mutants in this category, and the finding that each of these mutants binds to only a subset of the probes, these results fail to provide significant evidence that fingers 2 and 3 mediate a critical proteinprotein interaction.

\section{Homology modeling of fingers 2 and 3}

To examine potential structural roles for the critical DNA-binding residues revealed by the mutant analysis, a homology model was generated for fingers 2 and 3 using five, high-resolution crystal structures (Fig. 6) (ElrodErickson et al. 1998). Each of the structures had at least $50 \%$ sequence identity to fingers 2 and 3 of Ikaros. Models of reasonable accuracy have been created by homology modeling between proteins with approximately $50 \%$ sequence identity. These typically have a backbone RMS deviation of less than $1.0 \AA$ from the crystal structures determined after the models were produced (Chung and Subbiah 1996). Within a zinc finger, amino acids that commonly interact with the DNA are located at the -1 , 2,3 , and 6 positions in reference to the beginning of the $\alpha$ helix. Together, these residues define the surface that interacts with the major groove of the DNA, which in the models shown in Figure 6, would lie to the right, slightly angled above the surface of the page.

In finger 2 , the residues at the $-1,2,3$, and 6 positions are all important. Substitutions at the 3 and 6 positions (N159 and R162) completely disrupted both pericentromeric targeting and DNA binding. Substitutions at the -1 and 2 positions (Q156 and G158) disrupted targeting and severely diminished complex formation with five of the six probes. Most likely, G158 does not directly contact DNA; instead, this glycine may confer conformational flexibility to the peptide backbone.

In finger 3, the amino acids that commonly contact DNA do not appear to be as important as in finger 2 . Only the -1 position (R184) was critical for pericentromeric targeting and for DNA binding to all of the probes. Mutation of the 2 position (D186) severely reduced binding to four of the probes, but efficient binding to the consensus IK bs 4 probe was retained. Mutation of the 3 position (A187) severely reduced binding to two of the probes, including the consensus IK bs 4 probe. However, efficient binding was observed with the other four probes. Interestingly, the mutations that alter the 2 and 3 positions of finger 3 comprised two of the three mutants that exhibited variable DNA-binding properties coupled with pericentromeric targeting. Last, position 6 (G190) appeared to be unimportant for DNA binding or

\footnotetext{
Figure 5. Analysis of single amino acid substitutions within fingers 2 and 3 . (A) The sequences of fingers 2 and 3 are shown. As in Figure 4, the positions of the $\beta$ sheets and the $\alpha$ helixes are indicated, and the critical cysteines and histidines are underlined. An asterisk denotes the zinc finger positions that typically contact DNA (referred to as the $-1,2,3$, and 6 positions). The amino acid substitutions are shown below the sequence. Substitutions in red disrupted centromeric targeting, and those in blue did not. The effect of each substitution on DNA binding activity is indicated: A - indicates that the mutant failed to bind all of the probes, a + indicates that the mutant formed an abundant complex with 1-4 probes, and no notation indicates that the mutant formed an abundant complex with 5-6 probes. (B) The confocal images, obtained as described for the legend to Figure 1, are shown. The finger 2 mutants are shown at the top, and the finger 3 mutants, at the bottom. $(C)$ The DNA-binding activities of the mutant proteins were determined as described in the legend to Figure 2. A Western blot using HA antibodies (top panel) and the gel shift results obtained with six different probes (panels 2-7) are shown. The six probes were described in Figure 3 and in the legend to Figure 3.
} 
targeting, most likely because the amino acid at this position, a glycine, lacks a side-chain. In addition, the substitution of position 6 to an alanine did not create steric conflicts in the homology model.

The amino acids within fingers 2 and 3 that were critical for DNA binding were not restricted to the $-1,2$, 3 , and 6 positions. Three other amino acids appeared to be important for stabilizing the structure of the fingers. In finger 2, F154 appeared to be involved in packing the core of the zinc-finger homology model. However, the interacting partner from the $\alpha$-helix (L160) appeared to be unimportant. Perhaps, an adjacent leucine (L161) is capable of compensating for the substitution of L160 by taking its place in the zinc finger core. In finger 3, both of the hydrophobic residues predicted to be necessary for packing the zinc finger core (Y180 and L188) were critical for both DNA binding and pericentromeric targeting.

The roles of the three remaining amino acids that were important for DNA binding (K157, A181, and L192) could not be rationalized on the basis of the homology models. In finger 2, $\mathrm{K} 157$ (at the 1 position) is oriented away from the DNA. Perhaps this residue stabilizes the positions of adjacent residues or is involved in contacting the DNA-phosphodiester backbone. It also is possible that this lysine is incorrectly oriented in our homology model. In finger 3, A181 is located on the opposite side of the finger from the DNA binding surface. It is difficult to predict what structural effects substitutions would have at this position, but the substitution to a leucine might have resulted in the unfavorable positioning of a hydrophobic side-chain on the surface of the finger. Last, L192, which was important for binding to some of the gel shift probes but not for pericentromeric targeting, is located between two conserved histidines (H191 and H195), but is oriented toward the solvent away from the DNA.

Finally, the structural information discussed above is important to consider with respect to the correlation between DNA binding and pericentromeric targeting. Perhaps of greatest significance, five of the ten amino acids found to be essential for pericentromeric targeting mapped to the $-1,2,3$, or 6 positions that are typically critical for protein-DNA interactions (Q156, G158, N159, R162, and R184). Three other amino acids that were essential for pericentromeric targeting are required for packing the cores of the zinc fingers (F154, Y180, and L188). The remaining two amino acids involved in pericentromeric targeting (K157 and A181) are among those that do not have obvious roles; however, both of these amino acids are essential for stable binding to a subset of the probes. Overall, the concordance of the mutant data with the homology models substantially strengthens the hypothesis that Ikaros is targeted to centromeric foci on the basis of direct protein-DNA interactions.

\section{Discussion}

The results presented here provide strong evidence that a major mechanism by which Ikaros is targeted to foci of pericentromeric heterochromatin is through direct DNA binding. Consistent with the idea that pericentromeric targeting is an intrinsic property of Ikaros, targeting did not require lymphocyte-specific proteins or a lymphoid nuclear architecture. However, one important criterion for targeting was the capacity for dimer formation, as targeting was abolished by disruption of the carboxy-terminal dimerization domain.

It is important to note that the results do not rule out the possibility that protein-protein interactions contribute to pericentromeric targeting. Although an important protein-protein interaction surface was not identified, it remains possible that redundant protein-protein interactions are involved, with at least one interaction surface retained in each large deletion mutant. Our attempts to determine whether sequence-specific DNA binding is
Figure 6. Ribbon representation of the homology model of zinc fingers 2 and 3 . The amino acids that commonly interact with DNA $(-1,2,3$, and 6 positions in reference to the beginning of the $\alpha$ helix) are shown as numbered spheres on the gray backbone. The amino acids important for pericentromeric targeting or DNA binding are represented as ball-andstick diagrams. Residues in which a substitution disrupted both targeting and DNA binding to all of the gel shift probes are red. Residues in which a substitution disrupted targeting and DNA binding to a subset of the probes are yellow. Residues in which a substitution did not disrupt targeting but disrupted binding to a subset of the probes are blue. Models were generated using the programs MOLSCRIPT (Kraulis 1991) and RASTER3D (Merritt and Bacon 1997).
Finger 2

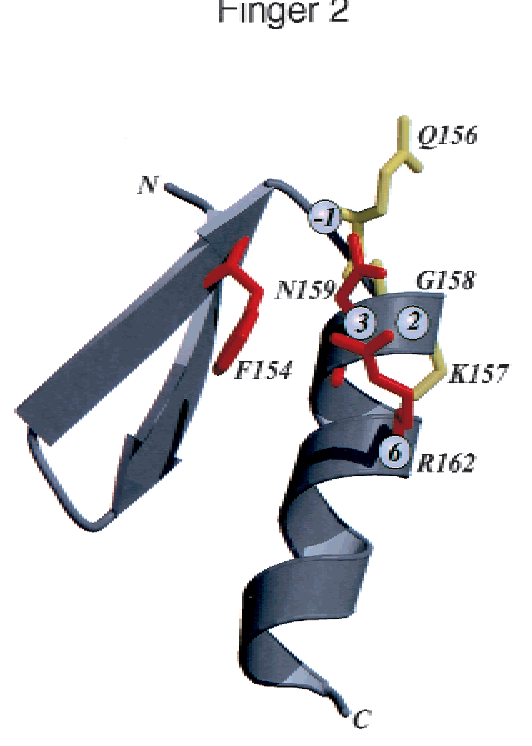

Finger 3

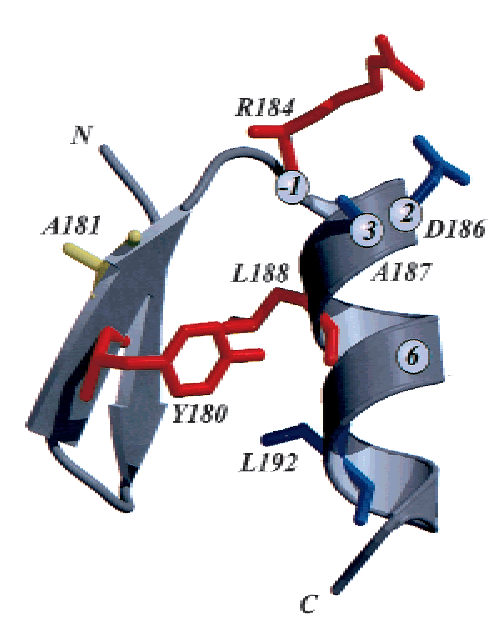


not only necessary, but also sufficient for targeting, have been hampered by protein stability problems. Nevertheless, the results presented here clearly show that pericentromeric targeting is absolutely dependent on the DNA-binding activity of Ikaros.

The finding that the DNA-binding domain of Ikaros is essential for pericentromeric targeting in fibroblasts strongly suggests that a similar targeting mechanism is used in lymphocytes. However, it is interesting to note that Ikaros is not efficiently localized to centromeric foci in resting lymphocytes and in some cycling lymphocytes (Brown et al. 1997; Hahm et al. 1998). In resting lymphocytes, Ikaros is distributed diffusely, but upon cell stimulation, it begins to form foci that migrate toward foci of pericentromeric heterochromatin (Brown et al. 1999). Because the centromeric foci were found to be intact in resting cells, the absence of Ikaros at these foci suggests that the subnuclear localization of Ikaros must be subject to regulation, perhaps by a posttranslational modification or by association with other proteins. The efficient pericentromeric targeting of overexpressed Ikaros in $3 \mathrm{~T} 3$ cells suggests that pericentromeric heterochromatin is the default site to which Ikaros dimers localize. In other words, unmodified Ikaros dimers may be targeted to centromeric foci, with a posttranslational modification or protein-protein interaction required for targeting to other regions of the nucleus.

The results obtained in this study raise two key questions. First, what DNA sequences are bound by Ikaros that result in pericentromeric targeting? Second, what models for the function of Ikaros at centromeric foci are consistent with these data? With respect to the Ikaros recognition sequences, the simplest hypothesis is that Ikaros binds directly to DNA elements within the centromeric repeats. Consistent with this hypothesis, the gel shift data show that centromeric repeats contain sites that are capable of binding Ikaros with a reasonably high affinity. Direct binding to centromeric repeats would provide an attractive explanation for the efficient targeting of the overexpressed Ikaros, as it is difficult to imagine efficient targeting of the overexpressed protein via binding to non-repetitive DNA sequences within pericentromeric heterochromatin. Additional experiments will be required to provide physical evidence of direct binding to centromeric repeats.

The evidence that Ikaros is targeted to centromeric foci via direct DNA binding rules out the simple hypothesis that the DNA-binding domain of Ikaros is dedicated to interactions with target genes. However, the data remain compatible with a revised version of this model. In this revised model (Fig. 7, model 1), Ikaros may exist as a multimer, with some subunits bound to centromeric repeats and others available for interactions with target genes. The possibility that Ikaros may exist in a multimeric state in lymphocytes was previously supported by gel filtration data (Hahm et al. 1998). However, using stringent conditions, the predominant Ikaros species detected in lymphocyte extracts appears to be a dimer (A. McCarty, B.S. Cobb, and S.T. Smale, unpubl.). Therefore, if Ikaros multimers exist in vivo, they most likely are
1. Targeting inactive genes to pericentromeric heterochromatin

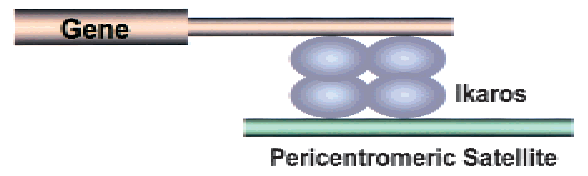

2. Targeting associated proteins to pericentromeric heterochromatin

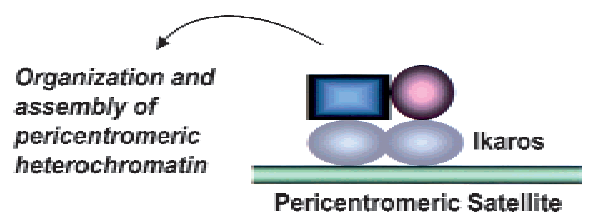

3. Storage at pericentromeric heterochromatin

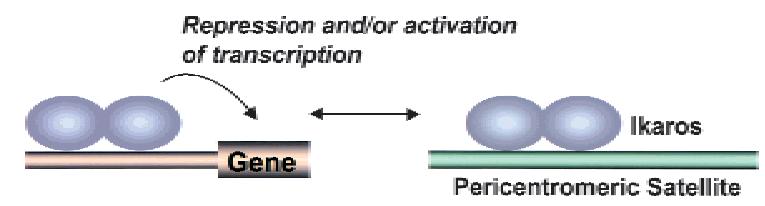

Figure 7. Models of Ikaros function at pericentromeric heterochromatin. (1) Ikaros may target specific genes to pericentromeric heterochromatin through multimerization, whereby some Ikaros subunits bind centromeric sequences and others bind sequences in specific target genes. (2) The Ikaros positioned at centromeric foci through sequence-specific DNA binding may recruit other proteins, like histone deacetylases, to the foci, thereby contributing to the organization and assembly of pericentromeric heterochromatin. (3) Centromeric foci may serve as a storage site for Ikaros, allowing rapid mobilization to other nuclear sites when its functions at those sites are required.

unstable and will be difficult to study in vitro. The preference for binding of monomeric Ikaros to the $\gamma$ sat B probe (Fig. 3) raises the alternative possibility that an Ikaros dimer binds two different DNA molecules simultaneously, with one subunit of the dimer bound to a centromeric repeat and the other bound to a site within a target gene. This possibility cannot be excluded. However, high-affinity binding to the $\mathrm{D}^{\prime}$ element of the TdT promoter, which is required for transcriptional downregulation during thymocyte development, requires promoter binding by both subunits of an Ikaros dimer (L.A. Trinh and S.T. Smale, unpubl.). Thus, it appears unlikely that a dimer will bind simultaneously to a centromeric repeat and a target gene.

Two alternative models that are equally consistent with the current data are diagrammed in Figure 7 (models 2 and 3). In model 2, the sole function of Ikaros at centromeric foci is to recruit other proteins to pericentromeric heterochromatin via protein-protein interactions. For example, a critical function of Ikaros could be to recruit the NURD histone deacetylase complex to centromeric foci, consistent with the finding that Ikaros 
and NURD colocalize to centromeric foci in lymphocytes (Kim et al. 1999). Once associated with the centromeric foci, the NURD complex could contribute to the deacetylation of centromeric DNA sequences or genes that are targeted to pericentromeric heterochromatin by other mechanisms. Such a function could allow Ikaros to contribute to gene regulation or the general organization and assembly of the centromeric foci. Alternatively, by recruiting other proteins to centromeric foci, Ikaros could contribute to the regulation of DNA replication or the maintenance of genome stability, as previously suggested (Avitahl et al. 1999). Why a tissue-specific DNAbinding protein would be involved in such fundamental processes is unclear, but perhaps other Ikaros family members perform similar roles in other mammalian cell types (Honma et al. 1999).

It is important to reconcile this second model with the proposal that Ikaros regulates the expression of target genes, like the TdT gene, by direct binding to sites within their promoters or enhancers. The simplest hypothesis would be that the function of Ikaros at centromeric foci is completely independent of its putative functions in relating target genes within euchromatic regions of the nucleus. This hypothesis is similar to that proposed by Csink and Henikoff (1998), who suggested that the assembly of pericentromeric heterochromatin may require functions supplied by transcription factors that typically function elsewhere in the nucleus. For example, a major function of Ikaros within euchromatin may be to recruit histone deacetylase complexes to target genes during transcriptional downregulation. Centromeric repeats may have acquired Ikaros binding sites during evolution because the assembly and organization of pericentromeric heterochromatin in lymphocytes may benefit from the use of these same complexes.

In model 3 (Fig. 7), the centromeric repeats serve as storage sites for Ikaros, with its important functions occurring elsewhere in the nucleus. The centromeric repeats may be an attractive location for the regulated storage of a protein, helping to sequester it from potential target genes at specific stages of development. For example, in the case of the TdT gene, some of the pericentromeric Ikaros (or Ikaros-Helios) complexes may be mobilized upon stimulation of double-positive thymocytes, allowing them to repress the $\mathrm{TdT}$ gene at non-centromeric sites.

In considering this third model, one important question that must be considered is whether the storage of Ikaros at centromeric foci is functionally relevant or a fortuitous event that may arise because the centromeric repeats happen to contain high-affinity binding sites. It is well established that the sequences of centromeric satellite repeats vary dramatically between species (Wiens and Sorger 1998). Therefore, the conservation of pericentromeric targeting through evolution would provide strong evidence that Ikaros performs an important function at these sites, as it is unlikely that high-affinity Ikaros binding sites would be conserved fortuitously. The published reports demonstrating that Ikaros localizes to phenotypically similar foci in trout and man
(Hansen et al. 1997; Sun et al. 1999) support the hypothesis that the targeting of Ikaros to pericentromeric heterochromatin is evolutionarily conserved and functionally important. In addition, it is noteworthy that human repeat sequences contain sites that are likely to bind Ikaros with high affinity (Choo et al. 1991). Nevertheless, attempts in our laboratory to confirm that Ikaros localizes to pericentromeric foci in other species have not yet been successful, possibly because pericentromeric heterochromatin is relatively easy to detect in murine cells.

The growing evidence that Ikaros binding to the TdT promoter is required for transcriptional downregulation during thymocyte development (L.A. Trinh and S.T. Smale, unpubl.), although at first glance inconsistent with the current results, is actually consistent with any of the three models. Intact Ikaros binding sites within the TdT promoter are essential for downregulation upon stimulation of a double-positive thymocyte line (L.A. Trinh and S.T. Smale, unpubl.), strongly suggesting that Ikaros directly contributes to downregulation. In some respects, model 1 is the least consistent with these data because Ikaros binding sites are important for $\mathrm{TdT}$ downregulation in a cell line in which the inactive TdT locus does not migrate to centromeric foci (Brown et al. 1999; L.A. Trinh and S.T. Smale, unpubl.). In contrast, the simplest version of model 1 implies that Ikaros would not be involved in the downregulation process until the locus moves to the centromeric foci. Nevertheless, a variation of model 1 would allow a dimer of Ikaros that is stably bound to the inactive, euchromatic TdT locus to associate with another dimer of Ikaros that is positioned at the centromeric foci, thereby resulting in repositioning of the locus. Models 2 and 3 are perhaps more consistent with the TdT data because the Ikaros that is initially involved in TdT downregulation would be independent of the pool of Ikaros positioned at the centromeric foci, although the binding of Ikaros to the TdT promoter could ultimately lead to repositioning of the TdT locus. Although the current data do not yield a complete understanding of the function of Ikaros at centromeric foci, the finding that DNA binding is a critical determinant of pericentromeric targeting provides a significant step toward this goal.

\section{Materials and methods}

Plasmids and retroviruses

Ikaros cDNAs were cloned into the mammalian expression vector pcDNA3 (InVitrogen). Each cDNA contained at the amino terminus an HA epitope tag, MYPYDVPDYAGGGLE. Deletions were prepared by PCR using primers corresponding to the end of the deletion. Deletions of the DNA-binding zinc fingers and all substitutions within those fingers were generated by a two step PCR sewing protocol. Constructs with the SV40 T Ag NLS contained the sequence PKKKRKV immediately downstream of the epitope tag. The construct with the leucine zipper at the carboxyl terminus contained Ikaros amino acids 1-458 fused to residues 244-315 of the Arabidopsis thaliana protein GBF1 (Schindler et al. 1992). 
For retrovirus generation, Ikaros cDNAs were cloned into the retroviral expression vectors pBABE puro (Morgenstern and Land 1990) or pMSCV pac (Hawley et al. 1994). These constructs were transfected into $293 \mathrm{~T}$ cells with the helper virus vector $\mathrm{p} \Psi$ eco using a calcium phosphate protocol. Culture supernatants containing the retrovirus particles were harvested 36,48 , and 60 hours post-transfection. For infection of NIH 3T3 cells, the virus supernatant was added to a sub-confluent plate of cells with $2 \mu \mathrm{g} / \mathrm{ml}$ polybrene, and the infection proceeded for 2-6 hours, after which the cells were fed with fresh media.

\section{Confocal microscopy}

For analysis of all Ikaros proteins, transduced NIH 3T3 cells were passaged onto cover slips 48 hours post-infection and were allowed to attach overnight. The cells were washed once in PBS, then fixed for $1 \mathrm{~min}$ in methanol on dry ice and permeabilized for 10 min in blocking buffer (PBS with $0.1 \%$ saponin and $10 \%$ fetal bovine serum). The cells were then incubated for $1 \mathrm{hr}$ with rabbit anti-Ikaros antibodies (either recognizing the amino-terminal or carboxy-terminal portions of Ikaros) (Hahm et al. 1994) diluted in blocking buffer. The cells were washed three times, 3 min each in PBS $0.1 \%$ saponin, then incubated for $1 \mathrm{hr}$ with an FITC-labeled goat anti-rabbit antibody diluted in blocking buffer. The cells were washed three times, 3 min each in PBS $0.1 \%$ saponin, one time in PBS, and mounted in vector shield containing $0.5 \mu \mathrm{g} / \mathrm{ml}$ propidium iodide.

For analysis of Ikaros and Mi-2, NIH 3T3 cells expressing Ikaros isoform VI were prepared as above. In the primary antibody incubation, a monoclonal anti-Ikaros antibody (2A9) (Hahm et al. 1998) was used along with a rabbit anti-Mi2 (CHD4-N), which was a gift of Weidong Wang, NIH (Xue et al. 1998). In the secondary antibody incubation, an FITC-labeled goat anti-rabbit antibody was used along with a Texas red-labeled goat anti-mouse IgG F(c). Cells were mounted in vector shield without propidium iodide.

The immunoFISH protocol was described previously (Brown et al. 1997). Confocal microscopy was done with a BioRad system using a Nikon microscope. For all images, the contrast was adjusted using Photoshop to enhance the indicated features.

\section{Nuclear extracts and gel shift assays}

Nuclear extracts were prepared as described (Lo et al. 1991) from 293 T cells transfected with the Ikaros constructs in the pcDNA3 expression vector. The relative amount of each Ikaros protein was determined in Western blots using the HA monoclonal antibody, 12CA5. The level of Ikaros protein was normalized by dilution, when necessary, with nuclear extract from cells transfected with vector alone. Gel mobility shift assays were performed as described (Lo et al. 1991). All probes were derived from synthetic double-stranded oligonucleotides. Each probe was designed to contain a $5^{\prime}$ overhang homologous to a Sall site, which facilitated labeling by filling in with Klenow and $\alpha-{ }^{32} \mathrm{P}$-labeled nucleotides. The sequences of the probes are shown in Figure 3B.

\section{Model building}

A homology model of zinc fingers 2 and 3 of Ikaros was generated using the SWISS MODEL protein modeling server (Peitsch 1996; Guex and Peitsch 1997). Five independently solved crystal structures that have at least $50 \%$ sequence identity to fingers 2 and 3 were used to generate the model. The model was visually inspected and obvious errors were corrected. Two independent methods were used to assess the quality of the homology model.
First, a ramachandran plot was calculated using the program, PROCHECK (Laskowski et al. 1993). The ramachandran plot shows the distribution of phi-psi angles for all residues in the structure. Of the residues in the Ikaros homology model, $84 \%$ were in the most favorable region, and only $2 \%$ fell into the disallowed region. Second, the crystal structure validation program, ERRAT, was used (Colovos and Yeates 1993). ERRAT compares the statistics of non-bonded atomic interactions for the model against a database of high-resolution crystal structures. Residues with unusual contact statistics are reported. No residues had unusual contact statistics, suggesting that the Ikaros homology model is accurate.

\section{Acknowledgments}

We thank Weidong Wang for providing the Mi-2 antibody, Steven Henikoff for valuable discussions, and Roger Ferrini, Aaron McCarty, Shomyseh Sanjabi, and Amy Weinmann for critical readings of the manuscript. We are also grateful to Sayeh Farivar for technical assistance. This work was supported by U.S. Public Health Service Grant R01 DK43726 (S.T. Smale) and by the Medical Research Council, U.K. (A.G.F.). S.T. Smale is an Associate Investigator with the Howard Hughes Medical Institute.

The publication costs of this article were defrayed in part by payment of page charges. This article must therefore be hereby marked "advertisement" in accordance with 18 USC section 1734 solely to indicate this fact.

\section{References}

Avitahl, N., Winandy, S., Friedrich, C., Jones, B., Yimin, G., and Georgopoulos, K. 1999. Ikaros sets thresholds for T cell activation and regulates chromosome propagation. Immunity 10: 333-343.

Brown, K.E., Baxter, J., Graf, D., Merkenschlager, M., and Fisher, A.G. 1999. Dynamic repositioning of genes in the nucleus of lymphocytes preparing for cell division. Mol. Cell 3: 207217.

Brown, K.E., Guest, S.S., Smale, S.T., Hahm, K., Merkenschlager, M., and Fisher, A.G. 1997. Association of transcriptionally silent genes with Ikaros complexes at centromeric heterochromatin. Cell 91: 845-854.

Choo, K.H., Vissel, B., Nagy, A., Earle, E., and Kalitsis, P. 1991. A survey of the genomic distribution of alpha satellite DNA on all the human chromosomes, and derivation of a new consensus sequence. Nucleic Acids Res. 19: 1179-1182.

Chung, S.Y. and Subbiah, S. 1996. A structural explanation for the twilight zone of protein sequence homology. Structure 4: 1123-1127.

Cobb, B. and Smale, S.T. 2000. Ikaros, in search of a molecular function during lymphocyte development. Curr. Top. Micro. Immun. (in press).

Colovos, C. and Yeates, T.O. 1993. Verification of protein structures: patterns of nonbonded atomic interactions. Protein Science 2: 1511-1519.

Cortes, M., Wong, E., Koipally, J., and Georgopoulos, K. 1999. Control of lymphocyte development by the Ikaros gene family. Curr. Opin. Immunol. 11: 167-171.

Csink, A.K. and Henikoff, S. 1998. Something from nothing: The evolution and utility of satellite repeats. Trends Genet. 14: 200-204.

Elrod-Erickson, M., Benson, T.E., and Pabo, C.O. 1998. Highresolution structures of variant Zif268-DNA complexes: im- 
plications for understanding zinc finger-DNA recognition. Structure 6: 451-464.

Glimcher, L.H. and Singh, H. 1999. Transcription factors in lymphocyte development- $\mathrm{T}$ and $\mathrm{B}$ cells get together. Cell 96: $13-23$.

Guex, N. and Peitsch, M.C. 1997. SWISS-MODEL and the Swiss-PdbViewer: An environment for comparative protein modeling. Electrophoresis 18: 2714-2723.

Hahm, K., Cobb, B.S., McCarty, A.S., Brown, K.E., Klug, C.A., Lee, R., Akashi, K., Weissman, I.L., Fisher, A.G., and Smale, S.T. 1998. Helios, a T cell-restricted Ikaros family member that quantitatively associates with Ikaros at centromeric heterochromatin. Genes \& Dev. 12: 782-796.

Hahm, K., Ernst, P., Lo, K., Kim, G.S., Turck, C., and Smale, S.T. 1994. The lymphoid transcription factor LyF-1 is encoded by specific, alternatively spliced mRNAs derived from the Ikaros gene. Mol. Cell. Biol. 14: 7111-7123.

Hansen, J.D., Strassburger, P., and Du Pasquier, L. 1997. Conservation of a master hematopoietic switch gene during vertebrate evolution: Isolation and characterization of Ikaros from teleost and amphibian species. Eur. I. Immunol. 27: 3049-3058.

Hawley, R.G., Lieu, F.H., Fong, A.Z., and Hawley, T.S. 1994 Versatile retroviral vectors for potential use in gene therapy. Gene Therapy 1: 136-138.

Honma, Y., Kiyosawa, H., Mori, T., Oguri, A., Nikaido, T., Kanazawa, K., Tojo, M., Takeda, J., Tanno, Y., Yokoya, S., et al. 1999. Eos: a novel member of the Ikaros gene family expressed predominantly in the developing nervous system. FEBS Lett. 447: 76-80.

Kim, J., Sif, S., Jones, B., Jackson, A., Koipally, J., Heller, E., Winandy, S., Viel, A., Sawyer, A., Ikeda, T., et al. 1999. Ikaros DNA-binding proteins direct formation of chromatin remodeling complexes in lymphocytes. Immunity 10: 345355.

Koipally, J., Renold, A., Kim, J., and Georgopoulos, K. 1999. Repression by Ikaros and Aiolos is mediated through histone deacetylase complexes. EMBO J. 18: 3090-3100.

Kraulis, P.J. 1991. MOLSCRIPT: A program to produce both detailed and schematic plots of protein structures. J. Appl. Cryst. 24: 946-950.

Laskowski, R.A., MacArthur, M.W., Moss, D.S., and Thornton, J.M. 1993. PROCHCECK: A program to check the stereochemical quality of protein structures. I. Appl. Cryst. 26: 283-291.

Lo, K., Landau, N.R., and Smale, S.T. 1991. LyF-1, a transcriptional regulator that interacts with a novel class of promoters for lymphocyte-specific genes. Mol. Cell. Biol. 11: 52295243.

Merritt, E.A. and Bacon, D.J. 1997. Raster 3D: Photorealistic molecular graphics. Methods Enzymol. 277: 505-524.

Molnár, A. and Georgopoulos, K. 1994. The Ikaros gene encodes a family of functionally diverse zinc finger DNA-binding proteins. Mol. Cell. Biol. 14: 8292-8303.

Morgenstern, J.P. and Land, H. 1990. Advanced mammalian gene transfer: High titre retroviral vectors with multiple drug selection markers and a complementary helper-free packaging cell line. Nucleic Acids Res. 18: 3587-3596.

Peitsch, M.C. 1996. ProMod and Swiss-Model: Internet-based tools for automated comparative protein modelling. Biochem. Soc. Trans. 24: 274-279.

Schindler, U., Menkens, A.E., Beckmann, H., Ecker, J.R., and Cashmore, A.R. 1992. Heterodimerization between lightregulated and ubiquitously expressed Arabidopsis GBF bZIP proteins. EMBO J. 11: 1261-1273.

Sun, L., Heerema, N., Crotty, L., Wu, X., Navara, C., Vassilev,
A., Sensel, M., Reaman, G.H., and Uckun, F.M. 1999. Expression of dominant-negative and mutant isoforms of the antileukemic transcription factor Ikaros in infant acute lymphoblastic leukemia. Proc. Natl. Acad. Sci. 96: 680-685.

Sun, L., Liu, A., and Georgopoulos, K. 1996. Zinc finger-mediated protein interactions modulate Ikaros activity, a molecular control of lymphocyte development. EMBO J. 15: 5358 5369.

Vissel, B. and Choo, K.H. 1989. Mouse major (gamma) satellite DNA is highly conserved and organized into extremely long tandem arrays: Implications for recombination between nonhomologous chromosomes. Genomics 5: 407-414.

Wiens, G.R. and Sorger, P.K. 1998. Centromeric chromatin and epigenetic effects in kinetochore assembly. Cell 93:313316.

Winandy, S., Wu, L., Wang, J.H., and Georgopoulos, K. 1999. Pre-T cell receptor (TCR) and TCR-controlled checkpoints in $\mathrm{T}$ cell differentiation are set by Ikaros. J. Exp. Med. 190: 1039-1048.

Wong, A.K. and Rattner, J.B. 1988. Sequence organization and cytological localization of the minor satellite of mouse. Nucleic Acids Res. 16: 11645-11661.

Xue, Y., Wong, J., Moreno, G.T., Young, M.K., Côté, J., and Wang, W. 1998. NURD, a novel complex with both ATPdependent chromatin-remodeling and histone deacetylase activities. Mol. Cell 2: 851-861. 


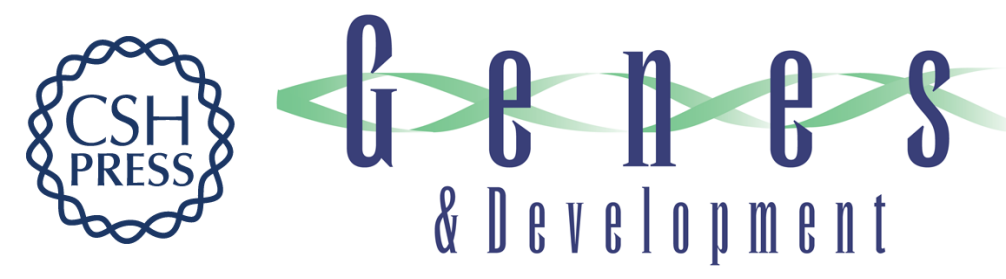

\section{Targeting of Ikaros to pericentromeric heterochromatin by direct DNA binding}

Bradley S. Cobb, Susana Morales-Alcelay, Gary Kleiger, et al.

Genes Dev. 2000, 14:

Access the most recent version at doi:10.1101/gad.816400

References This article cites 31 articles, 8 of which can be accessed free at: http://genesdev.cshlp.org/content/14/17/2146.full.html\#ref-list-1

License

Email Alerting

Receive free email alerts when new articles cite this article - sign up in the box at the top Service right corner of the article or click here.

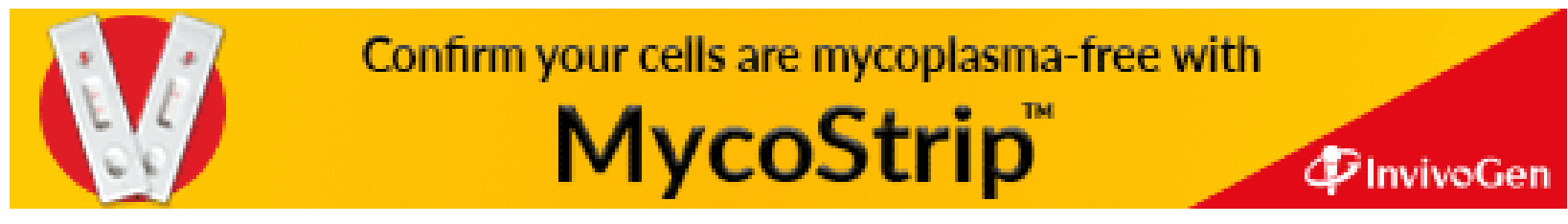

